\title{
Multiple-well tunneling model for the magnetic-field effect in ultracold glasses
}

\author{
Giancarlo Jug* \\ Dipartimento di Fisica e Matematica, Università dell'Insubria, Via Valleggio 11, 22100 Como, Italy \\ CNISM-Unità di Ricerca di Como and INFN-Sezione di Pavia, Italy
}

(Received 16 April 2009; published 8 May 2009)

\begin{abstract}
Puzzling observations of unusual responses of some multisilicate glasses at temperatures $T<1 \mathrm{~K}$ to static magnetic fields $B$ have been reported in the last decade and call for an extension of the standard two-level systems tunneling model. An explanation is proposed capable of capturing at the same time the $T$ and $B$ dependences of the heat capacity $C_{p}$ and of the dielectric constant $\epsilon$ in these glasses. This theory points to the existence of anomalous multiwelled tunneling systems in the glasses-alongside the standard two-level systems - and indications are given for glasses which should achieve much larger electric magnetocapacitive enhancements.
\end{abstract}

DOI: 10.1103/PhysRevB.79.180201

PACS number(s): 64.70.ph, 61.43.Fs, 65.60.+a, 77.22.Ch

The last decade has seen much renewed interest in the physics of cold nonmetallic glasses, materials displaying some quasiuniversal physical properties attributed to the low-energy excitations characterizing all amorphous solids. The two-level system (2LS) tunneling model (TM) (Ref. 1) has been rather successful in explaining a variety of thermal, dielectric, and acoustic anomalies in structural glasses at temperatures $T<1 \mathrm{~K}$. Limitations and failures of the $2 \mathrm{LS}$ TM (treating cooperative phenomena in terms of single particles, mostly), on the other hand, have also been discussed. ${ }^{2}$

These materials do not present, normally, any remarkable magnetic-field response phenomena other than possibly a weak contribution from trace paramagnetic Fe impurities. It came thus as a great surprise when some measurements showed $^{3}$ that in some multicomponent silicate glasses (but not in pure silica), one is able to observe changes in the dielectric constant $\epsilon(T, B)$ and already in magnetic fields $B$ as weak as a few Oe. A typical glass showing a strong response has some $100 \mathrm{ppm} \mathrm{Fe}^{3+}$ and the composition $\mathrm{Al}_{2} \mathrm{O}_{3}-\mathrm{BaO}-\mathrm{SiO}_{2}$ (in short $\mathrm{AlBaSi}-\mathrm{O}$, in this paper). Measurements made on a thick sol-gel fabricated film showed changes in $\delta \epsilon / \epsilon=[\epsilon(B)-\epsilon(0)] / \epsilon(0)$ on the order $10^{-4}$ and characterized by an enhancement peaking around $0.03 \mathrm{~T}$ for $10 \mathrm{mK}<\mathrm{T}<200 \mathrm{mK}$ then followed by a reduction of $\epsilon$ for $B>0.1$ T. A further enhancement was also observed at much higher fields $(B>10 \mathrm{~T})$.

Another-cleaner-multicomponent silicate glass (borosilicate BK7, with 6 ppm $\mathrm{Fe}^{3+}$ ) and a dirtier one (borosilicate Duran, with $120 \mathrm{ppm} \mathrm{Fe}^{3+}$ ) have shown similar-but weaker-magnetic anomalies of about $10^{-5}$, seemingly excluding the paramagnetic impurities as their source. ${ }^{4}$ Yet another multisilicate glass, $a-\mathrm{SiO}_{2+x} \mathrm{C}_{y} \mathrm{H}_{z}$ was investigated, ${ }^{5}$ confirming the unusual findings in AlBaSi-O. A convincing explanation for this unusual magnetocapacitive behavior has not yet been found.

Recently, some consensus has been gained by the idea of a coupling of the standard 2LS to the magnetic field via nuclei in the glasses carrying an electric-quadrupole moment, as well as a magnetic-dipole one. ${ }^{6}$ The nuclear mechanism was suggested by some features of the observed magnetic-field dependence of the polarization echo (PE) experiment in the mentioned multisilicate glasses in the millikelvin range. ${ }^{7}$ Moreover, the amplitude of the PE in glyc- erol glass was shown to become strongly $B$ dependent only upon deuteration and thus the introduction of quadrupolemoment carrying nuclei in the glass. ${ }^{8}$ However, though pure $a-\mathrm{SiO}_{2}$ (devoid of quadrupole-moment carrying nuclei) shows no PE-amplitude magnetic-field dependence, ${ }^{7}$ glycerol glass-deuterated or not-shows no measurable $B$ dependence in its dielectric constant. ${ }^{9}$ The nuclear approach is as yet unable to account for the magnitude and features of the $B$ and $T$ dependences of $\delta \epsilon / \epsilon$ for the multisilicate glasses ${ }^{10}$ and the (also unusual) $B$ and $T$ dependences of the heat capacity $C_{p}$ of the latter glasses-not entirely linked to their Fe impurity contents ${ }^{11,12}$ - has not yet been addressed. To add to the mystery, the acoustic response (also linked to the 2LS coupling to phonons) of borosilicate glasses BK7 and AF45 has been found to be independent of $B .{ }^{13}$ Table I summarizes this rather puzzling experimental situation. Therefore, either the nuclear explanation is specific to the $\mathrm{PE}$ magnetic effect or an entirely different explanation needs to be found for all of the observations.

The purpose of this Rapid Communication is to begin to give a rationale to the situation in Table $\mathrm{I}$, as well as to stimulate further experimental and theoretical research. An explanation, already shown to account for the unusual behavior of $C_{p}(T, B)$ in the multisilicate glasses, ${ }^{12}$ is here shown to explain the behavior of the dielectric constant as well. This simple theory, stemming from Ref. 14, is centered on the observation-in computer simulations and experiments ${ }^{15}$ - that multisilicate glasses present in their atomic structure both the connected network of $\mathrm{SiO}_{4}$ tetrahedra and a collection of "pockets and channels" of non-networking ions showing a tendency to form microaggregates and to partially destroy the $\mathrm{SiO}_{4}$ network. Figure 4 of Ref. 15 shows as an example a snapshot of a simulation of the $\left(\mathrm{Na}_{2} \mathrm{O}\right)-3\left(\mathrm{SiO}_{2}\right)$ glass illustrating such situation. The present theory proposes that the magnetic effects arise (at least for the multisilicates) from anomalous tunneling systems (ATS) forming in the cooling of such structure within the nonnetworking [or network-modifying (NM)] pockets and channels, while the $\mathrm{SiO}_{4}$ network remains the nest of the ordinary nonmagnetic $2 \mathrm{LS}$.

In order to couple the ATS to the magnetic field, a simple three-dimensional (3D) generalization of the 2LS TM is in order. As is known, ${ }^{1}$ in the TM the cold glass is thought to 
TABLE I. Presence of magnetic-field induced variations in the physical properties of some cold glasses. $C_{p}$ : heat capacity, $\epsilon$ : dielectric constant, $v_{s}$ : sound velocity, and $A_{\mathrm{PE}}$ : PE amplitude (?: no investigation known).

\begin{tabular}{lccccc}
\hline \hline Glass type & Ref. & $\delta C_{p}$ & $\delta \epsilon$ & $\delta v_{s}$ & $\delta A_{\mathrm{PE}}$ \\
\hline$a-\mathrm{SiO}_{2}$ & 11,4 , and 7 & NO & NO & $?$ & NO \\
$a-\mathrm{SiO}_{2+x} \mathrm{C}_{y} \mathrm{H}_{z}$ & 5 & $?$ & YES & $?$ & $?$ \\
$\mathrm{AlBaSi}-\mathrm{O}$ & 11,3 , and 7 & YES & YES & $?$ & YES \\
Duran & 11,4 , and 7 & YES & Weak & $?$ & YES \\
$\mathrm{BK} 7$ & $11,4,13$, and 7 & weak & Weak & NO & YES \\
$\mathrm{AF} 45$ & 13 & $?$ & $?$ & NO & $?$ \\
Glycerol & 9 and 8 & $?$ & NO & $?$ & Weak \\
$d$-Glycerol & 9 and 8 & $?$ & NO & $?$ & YES \\
\hline \hline
\end{tabular}

have few remaining degrees of freedom represented by fictitious "particles," each moving quantum mechanically within a one-dimensional (1D) double-welled potential. Only the ground states of the individual wells $|i\rangle(i=1,2)$ are relevant and in this representation the Hamiltonian of a single 2LS reads as $H_{0}=-\frac{1}{2} \Delta \sigma_{z}-\frac{1}{2} \Delta_{0} \sigma_{x}\left(\sigma_{\mu}\right.$ Pauli matrices) with $\Delta$ as the ground-state energy asymmetry between the two wells and $\Delta_{0}$ is the barrier's transparency. These two parameters are taken, normally, to be distributed in the glass so that $\Delta$ and $\ln \Delta_{0}$ (roughly, the potential barrier) have a uniform distribution $\mathcal{P}_{2 \mathrm{LS}}\left(\Delta, \Delta_{0}\right)=\bar{P} / \Delta_{0}, \bar{P}$ being a material-dependent constant. This description holds for the network-forming (NF) TS. For the NM ATS, instead, the simplest 3D generalization of the 2LS TM is that of other fictitious charged particles, each moving in a multiwelled 3D potential (see Fig. 1) and coupling to the magnetic field through their orbital motion. For the simplest case of $n_{w}=3$ potential wells, one can use

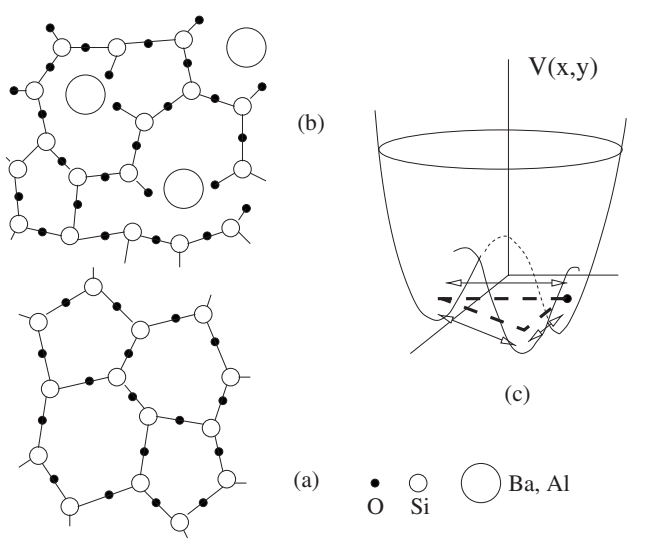

FIG. 1. 2D representation of the plausible source of magneticfield sensitive (anomalous) tunneling systems in (e.g.) the AlBaSi-O glass. (a) The tight vitreous- $\mathrm{SiO}_{2}$ structure is broken up by the (b) $\mathrm{Al}$ and (large) $\mathrm{Ba}$ atoms, thus leaving many metal ions free to move as "particles" in a 3D tunneling potential characterized by $n_{w}$ minima, with (c) $n_{w} \geq 3$.

$$
H_{0}=\left(\begin{array}{ccc}
E_{1} & D_{0} e^{i \phi / 3} & D_{0} e^{-i \phi / 3} \\
D_{0} e^{-i \phi / 3} & E_{2} & D_{0} e^{i \phi / 3} \\
D_{0} e^{i \phi / 3} & D_{0} e^{-i \phi / 3} & E_{3}
\end{array}\right),
$$

where $D_{0}$ is some $3 \mathrm{D}$ barrier's transparency, $E_{1}, E_{2}, E_{3}$ are the single wells' ground-state energy asymmetries, and

$$
\begin{gathered}
\phi=2 \pi \Phi(\mathbf{B}) / \Phi_{0} \\
\Phi(\mathbf{B})=\mathbf{B} \cdot \mathbf{S}_{\Delta}=B S_{\Delta} \cos \beta
\end{gathered}
$$

is the Aharonov-Bohm phase for a particle carrying charge $q$ tracing a closed path of area $S_{\Delta}$ threaded by a magnetic flux $\Phi(\mathbf{B})\left(\Phi_{0} \equiv h /|q|=\varphi_{0}|e / q|\right.$ being the appropriate flux quantum, $\varphi_{0}$ is the elementary one).

In this model, the choice $D_{0}>0$ can be thought of as arising, ultimately, from the coherent tunneling motion of a small cluster of NM ions; this will lead, as is seen, to high values of the product $|q| S_{\Delta} D_{0}$. For $D \equiv \sqrt{E_{1}^{2}+E_{2}^{2}+E_{3}^{2}} \ll D_{0}$ and weak fields $(\phi \ll 1)$, the lowest-energy gap of the model is seen to open with increasing magnetic field according to the simplified expression ${ }^{12} \Delta \mathcal{E} \simeq \sqrt{D_{0}^{2} \phi^{2}+D^{2}}$ containing the main physics of this model (regardless of the value of $n_{w}$ ). One more assumption of this theory (explaining the nearly flat $T$ dependence of $C_{p}$ at $B=0$ in some temperature range for these glasses ${ }^{12}$ ) is to take the ATS parameters' distribution uniform for $\ln D_{0}$ but favoring near degeneracy (to a degree fixed by a lower bound $D_{\min }>0$ for $D$ ) for the $\left\{E_{i}\right\}$,

$$
\mathcal{P}_{\text {ATS }}\left(\left\{E_{i}\right\}, \cdots ; D_{0}\right)=\frac{P^{*}}{\left(E_{1}^{2}+E_{2}^{2}+E_{3}^{2}+\cdots\right) D_{0}} .
$$

This anomalous distribution for the ATS can be thought of as arising from a degree of devitrification in the materials, as measured by the parameter $P^{*}$. In fact, it is reported ${ }^{16}$ that thick glass films prepared with the sol-gel technique (such as the AlBaSi-O films of the experiments) are in truth multiphase materials with microcrystals embedded within an amorphous glassy matrix. ${ }^{17,18}$ Indeed, amorphous solids with the general composition $\mathrm{Al}_{2} \mathrm{O}_{3}-\mathrm{MgO}-\mathrm{CaO}-\mathrm{SiO}_{2}$ are termed "glass ceramics" in the literature ${ }^{17}$ owing to occurring partial devitrification. It seems thus reasonable to imagine that in 
these glasses some NM ions provide nucleation centers for the microcrystals and that, therefore, TS presenting nearsymmetric wells will be favored in the neighborhood of and inside such crystallites.

This approach has provided a very good description ${ }^{12}$ of the $C_{p}(T, B)$ data for AlBaSi-O and Duran; ${ }^{11}$ one can treat the ATS as effective 2LS having gap $\Delta \mathcal{E}$ for "weak" fields. Within this picture, the linear-response quasistatic resonant contribution to the polarizability is

$$
\alpha_{\mu \nu}^{\mathrm{RES}}=\int_{0}^{\infty} \frac{d E}{2 E} \mathcal{G}_{\mu \nu}\left(\left\{\frac{E_{i}}{E}\right\} ; \mathbf{p}_{i}\right) \tanh \left(\frac{E}{2 k_{B} T}\right) \delta(E-\Delta \mathcal{E}),
$$

where

$$
\mathcal{G}_{\mu \nu}\left(\left\{\frac{E_{i}}{E}\right\} ; \mathbf{p}_{i}\right)=\sum_{i=1}^{n_{w}} p_{i \mu} p_{i \nu}-\sum_{i, j} \frac{E_{i} E_{j}}{E^{2}} p_{i \mu} p_{j \nu}
$$

contains the single-well dipoles $\mathbf{p}_{i}=q \mathbf{a}_{i}$. This expression assumes vanishing electric fields and no TS-TS interactions: a situation which does not wholly apply to the experiments. To keep the theory simple one can still use Eq. (4) and the analogous one for the relaxational contribution to the polarizability. Equation (4) must be averaged over the random energies' distribution (3) $\left([\ldots]_{a v}\right.$, responsible for the high sensitivity to weak fields) and over the dipoles' orientations and strengths $\overline{[(\ldots)]}$. For a collection of ATS with $n_{w}>2$, this averaging presents serious difficulties and one must resort to the decoupling,

$$
\overline{\mathcal{G}_{\mu \nu} \delta(E-\Delta \mathcal{E})} \simeq \overline{\mathcal{G}_{\mu \nu}} \cdot \overline{\delta(E-\Delta \mathcal{E})},
$$

where $\overline{[\delta(E-\Delta \mathcal{E})]_{\mathrm{av}}}=g_{\mathrm{ATS}}(E, B)$ is the fully averaged density of states. To calculate $\mathcal{G}_{\mu \nu}$, one can envisage a fully isotropic distribution of planar $n_{w}$ polygons to obtain

$$
\overline{\mathcal{G}_{\mu \nu}}=\frac{1}{3}\left(\frac{n_{w}}{n_{w}-1}\right) \overline{p_{i}^{2}} \frac{\left(n_{w}-2\right) E^{2}+D_{0}^{2} \phi^{2}}{E^{2}} \delta_{\mu \nu} .
$$

The second term in the numerator of Eq. (7) gives rise to a peak in $\delta \epsilon / \epsilon$ at very low $B$, while the first term (present only if $n_{w}>2$ ) gives rise to a negative contribution to $\delta \epsilon / \epsilon$ at larger $B$ which can win over the enhancement term for all values of $B$ if $D_{0 \text { max }} \gg D_{0 \text { min }}\left(D_{0 \text { min }}, D_{0 \text { max }}\right.$ corresponding to cutoffs in the distribution of ATS energy barriers). The observations in Duran and BK7 indeed show a significant depression of $\epsilon(B)$ for weak fields, ${ }^{4}$ thus giving direct evidence for the existence of ATS with $n_{w}>2$ in the multisilicate glasses.

Figure 2 shows the change due to a magnetic field in the dielectric constant $\epsilon(T, B)=\epsilon_{0}+\alpha(T, B)$ for the AlBaSi-O glass within the present oversimplified theory, which is used to fit the data at $118 \mathrm{mK} .^{4}$ It is seen that using the parameters extracted from fitting this theory ${ }^{12}$ to the $C_{p}(T, B)$ data $^{11}$ and a value $A=20.0 \times 10^{-5}$ for the dimensionless prefactor $A \equiv \overline{p_{i}^{2}} P^{*} /\left(\epsilon_{0} \epsilon_{r} D_{\min }\right)$, one can reproduce most of the qualitative features of the puzzling $B$ dependence of the magnetocapacitance for this glass. A best fit to the $118 \mathrm{mK}$ experimental data yields the parameters reported in Table II. The $T$ dependence of the calculated magnetocapacitance is also in

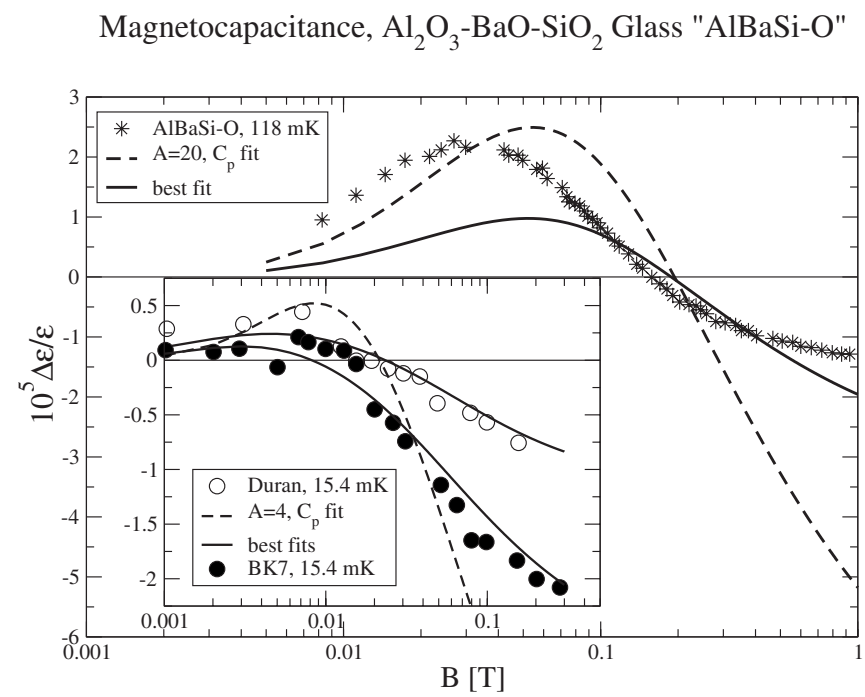

FIG. 2. Dielectric constant's change (real part) in a magnetic field, measured and from the present theory, for the multicomponent AlBaSi-O glass at $T=118 \mathrm{mK}$ (driving field: $15 \mathrm{kV} \mathrm{m}^{-1}$; driving frequency: $1 \mathrm{kHz}$ ) (inset: data and theoretical calculations for the BK7 and Duran glasses at $15.4 \mathrm{mK}$ ). Data are from Ref. 4.

qualitative agreement with the experimental data., ${ }^{3,4}$ In a comparison with data for the BK7 and Duran glasses ${ }^{4}$ (at the much lower only available temperature of $15.4 \mathrm{mK}$, however), the present theory reproduces the main features correctly. The values of $D_{0 \min }|q / e| S_{\Delta}$ and $D_{0 \max }|q / e| S_{\Delta}$ are very similar to those extracted from the $C_{p}(T, B)$ data; ${ }^{12}$ the small values of $D_{0 \min } / D_{0 \text { max }}$ and of $A$ for BK7 denote a much reduced presence of microcrystallites in that material. The values of $D_{\min }$ are lower (due to the strong electric field applied) and more realistic than those used in the analysis of $C_{p}(T, B)$, in all cases confirming the consistency of the assumption $\left|E_{i}\right| / D_{0} \ll 1$ in this theory.

Finally, the present simplified theory has focused on the weak $B$-field regime (up to $1 \mathrm{~T}$ ). Given the large values of $D_{0}|q / e| S_{\Delta}$ thus extracted (indicating that small coherent clusters of some 5 to $10 \mathrm{NM}$ ions are involved in the magneticsensitive tunneling ${ }^{13}$ ), one can expect the low- $B$ expression employed for the gap to break down around some larger field $B^{*} \sim \varphi_{0}\left(D_{0 \min } / D_{0 \max }\right) /\left(2 \pi|q / e| S_{\Delta}\right)$ above which the gap grows sublinearly with $\phi(B)$. As will be shown elsewhere, this is in turn responsible for the second enhancement of $\delta \epsilon / \epsilon$ observed in the experiments for $B>B^{*}$.

In summary, the ingredients of the present two-species TM, together with the reasonable assumption of partial devitrification in the films (which can be checked through X-ray

TABLE II. Local ATS parameters extracted from this fit.

\begin{tabular}{lcccc}
\hline \hline $\begin{array}{l}\text { Glass } \\
\text { type }\end{array}$ & $A$ & $\begin{array}{c}D_{\min } \\
(\mathrm{mK})\end{array}$ & $\begin{array}{c}D_{0 \min }|q / e| S_{\Delta} \\
\left(\mathrm{K} \mathrm{\AA} \AA^{2}\right)\end{array}$ & $\begin{array}{c}D_{0 \max }|q / e| S_{\Delta} \\
\left(\mathrm{K} \mathrm{\AA}^{2}\right)\end{array}$ \\
\hline AlBaSi-O & $1.42 \times 10^{-4}$ & 30 & $9.72 \times 10^{4}$ & $1.69 \times 10^{5}$ \\
Duran & $0.98 \times 10^{-5}$ & 3.4 & $1.83 \times 10^{4}$ & $6.28 \times 10^{5}$ \\
BK7 & $0.95 \times 10^{-5}$ & 24 & $4.16 \times 10^{4}$ & $1.09 \times 10^{6}$ \\
\hline \hline
\end{tabular}


analysis) allow for a good understanding of the puzzle of the magnetocapacitance in the cold multisilicate glasses. The absence of a magnetoacoustic response in such glasses with ATS can be understood from the much higher resonance frequencies of the NM pockets and channels and experiments should be done in such conditions. As for the PE experiments, an orbital-coupling approach with the inclusion of TS-TS interactions has been shown to provide a partial explanation for the $B$ dependence of the PE amplitude at ultralow temperatures. ${ }^{19}$ The present theory-interaction improved - might also provide an explanation for some such data, although the isotope effect ${ }^{8}$ makes the nuclear mecha- nism definitely the most promising. Further experiments are needed. Clearly, if the magnetic effects are due to quadrupole moments then the response should scale with the quadrupole-carrying nuclear concentration. If tunneling paramagnetic moments are involved, as suggested by a localized TM also capable of providing a good explanation for the $C_{p}$ and $\epsilon$ data $^{20}$ then the magnetic response should scale with the Fe concentration. In the present approach, the response scales with the NM ions' concentration and with the degree of devitrification; thus a larger magnetic response than thus far observed should be found in the best ceramic glasses, for instance, Ceran. *giancarlo.jug@uninsubria.it

${ }^{1}$ W. A. Phillips, J. Low Temp. Phys. 7, 351 (1972); P. W. Anderson, B. I. Halperin, and C. M. Varma, Philos. Mag. 25, 1 (1972); Tunneling Systems in Amorphous and Crystalline Solids, edited by P. Esquinazi (Springer-Verlag, Berlin, 1998).

${ }^{2}$ C. C. Yu and A. J. Leggett, Comments Condens. Matter Phys. 14, 231 (1988)

${ }^{3}$ P. Strehlow, C. Enss, and S. Hunklinger, Phys. Rev. Lett. 80, 5361 (1998); P. Strehlow, M. Wohlfahrt, A. G. M. Jansen, R. Haueisen, G. Weiss, C. Enss, and S. Hunklinger, ibid. 84, 1938 (2000).

${ }^{4}$ M. Wohlfahrt, P. Strehlow, C. Enss, and S. Hunklinger, Europhys. Lett. 56, 690 (2001); M. Wohlfahrt, Ph.D. thesis, Kirchhoff Institut für Physik, Universität at Heidelberg, Heidelberg, 2001; www.ub.uni-heidelberg.de/archiv/1587

${ }^{5}$ J. Le Cochec, F. Ladieu, and P. Pari, Phys. Rev. B 66, 064203 (2002).

${ }^{6}$ A. Würger, A. Fleischmann, and C. Enss, Phys. Rev. Lett. 89, 237601 (2002).

${ }^{7}$ S. Ludwig, C. Enss, P. Strehlow, and S. Hunklinger, Phys. Rev. Lett. 88, 075501 (2002); S. Ludwig, P. Nagel, S. Hunklinger, and C. Enss, J. Low Temp. Phys. 131, 89 (2003).

${ }^{8}$ P. Nagel, A. Fleischmann, S. Hunklinger, and C. Enss, Phys. Rev. Lett. 92, 245511 (2004).
${ }^{9}$ M. Brandt, Ph.D. thesis, Kirchhoff Institut für Physik, Universität at Heidelberg, Heidelberg, 2004; http://www.ub.uniheidelberg.de/archiv/4822

${ }^{10}$ D. Bodea and A. Würger, J. Low Temp. Phys. 136, 39 (2004).

${ }^{11}$ L. Siebert, Ph.D. thesis, Kirchhoff Institut für Physik, Universität at Heidelberg, Heidelberg, 2001; www.ub.uni-heidelberg.de/ archiv/1601

${ }^{12}$ G. Jug, Philos. Mag. 84, 3599 (2004).

${ }^{13}$ M. Layer, M. Heitz, J. Classen, C. Enss, and S. Hunklinger, J. Low Temp. Phys. 124, 419 (2001).

${ }^{14}$ S. Kettemann, P. Fulde, and P. Strehlow, Phys. Rev. Lett. 83, 4325 (1999).

${ }^{15}$ A. Meyer, J. Horbach, W. Kob, F. Kargl, and H. Schober, Phys. Rev. Lett. 93, 027801 (2004).

${ }^{16}$ G. Schuster, G. Hechtfischer, D. Buck, and W. Hoffmann, Rep. Prog. Phys. 57, 187 (1994).

${ }^{17} \mathrm{H}$. Bach and D. Krause, Analysis of the Composition and Structure of Glass and Glass Ceramics (Springer, New York, 1999).

${ }^{18}$ N. Pellegri, E. J. C. Dawnay, and E. M. Yeatman, J. Sol-Gel Sci. Technol. 13, 783 (1998); See, e.g., G. De et al., J. Non-Cryst. Solids 194, 225 (1996); X. L. Duan et al., J. Cryst. Growth 252, 311 (2003)

${ }^{19}$ A. Akbari and A. Langari, Phys. Rev. B 72, 024203 (2005).

${ }^{20}$ A. Borisenko and A. Bakai, Physica B 388, 112 (2007). 